\title{
Germanica
}

\section{Gespräch mit Falk Richter. Europäisches Theater heute}

Interview with Falk Richter: Today's European Theatre

Entretien avec Falk Richter : Théâtre européen d'aujourd'hui

\section{Nadia Foisil}

\section{OpenEdition}

Journals

Édition électronique

URL : http://journals.openedition.org/germanica/2552

DOI : $10.4000 /$ germanica. 2552

ISSN : 2107-0784

\section{Éditeur}

Université de Lille

\section{Édition imprimée}

Date de publication : 30 juin 2014

Pagination : 93-105

ISBN : 9782913857339

ISSN : 0984-2632

Référence électronique

Nadia Foisil, « Gespräch mit Falk Richter. Europäisches Theater heute », Germanica [Online], 54 | 2014 Online erschienen am: 30 Juni 2017, abgerufen am 06 Oktober 2020. URL : http://

journals.openedition.org/germanica/2552 ; DOI : https://doi.org/10.4000/germanica.2552 


\title{
Gespräch mit Falk Richter. Europäisches Theater heute
}

\author{
Nadia FoISIL \\ Université de Strasbourg-Freie Universität Berlin
}

Der Schwerpunkt unserer Recherche, der Körper, hat uns dazu geführt, uns mit der Opposition zwischen den psychologischen und den physischen Ansätzen im beruflich erlernenden Milieu zu befassen. Im Laufe dieser Forschungsjahre und durch die Hin- und Rückreise zwischen Theorie und Praxis genauso wie Ländern sind verschiedene Fragen entstanden. Inwieweit ist dieser Widerstand relevant bezüglich der Frage des Körpers und in welchem Sinne ist diese Dichotomie des Psychologischen und des Physischen mit der, die im Rahmen der Beziehung zwischen dem Text und dem postdramatischen Theater stattfindet, verbunden?

Im Kontext dieser Forschung (Doktorarbeit) haben wir uns bemüht, eine Theater-Anthropologie des Schauspielerkörpers als Instrument zu entwickeln, die aus theoretischen und empirischen Recherchen besteht und sich an verschiedene Fachbereiche, wie erstens Anthropologie und Theater, aber auch Soziologie, Paläontologie, Anatomie, Neurologie und Philosophie richtet. Unser Forschungsgebiet hat sich durch Beobachtungen und Teilnahme in Frankreich und Deutschland unter anderem an der Theaterschule Cours Florent (Paris, 2007-2008), am Laboratoire d'Étude du Mouvement (Studio der Bewegungsforschung) der Internationalen Theaterschule Jacques Lecoq (Paris, 2008-2009), sowie am Mime Centrum Berlin (2010-2012) und in verschiedenen Workshops herausgebildet. Qualitative Interviews gehören zu unserem Forschungsdesign und unterstützen auch die formulierten Hypothesen, 
nach welchen Natur und Kultur nicht gegensätzlich sind und beide zusammen zum Talentausdruck beitragen können (Problematik der Doktorarbeitsforschung). Diese Interviews wurden mit Körperfachleuten aus verschiedenen wissenschaftlichen und künstlerischen Bereichen (19 Interviews) und auch mit mehr als dreißig Lernenden der Bildungsinstitutionen, in denen die Beobachtung und Teilnahme stattfanden, geführt. Hinzu kommt eine Erweiterung der Erfahrungen in Köpertechniken durch regelmäßige Teilnahme an Workshops in Solo Performance, Installation, Körper und Stimme (Anke Gerber, Andrew Morrish, Sten Rudstrom, Jo Tomalin, Brenda Waite in Berlin, Teatro Punto in Italien). Unser Engagement als Zuschauer in Frankreich und in Deutschland hat auch folgende Frage hervorgehoben: Um welches Theater handelt es sich? Was verbindet Schiller, Aischylos, Racine, Pommerat und Richter? Die Vielfalt der Theaterformen ist nämlich umfangreich. Deshalb haben wir uns entschieden, uns auf eine besondere Art des Theaters zu fokussieren. Zu den Fachleuten, die wir interviewt haben, gehört auch Falk Richter. Mit folgenden Schwerpunkten werden wir sein Werk und seine Meinung über das Theater aufgreifen: Die Verbindung zwischen Text und Performativität, das Thema des Autors/Regisseurs und des zeitgenössischen Körpers und schließlich die Rolle des Zuschauers.

\section{Text und Performativität}

Körper und Text sind die Grundelemente des Theaters. Im Laufe der Zeit haben sich die Formen des Theaters entwickelt, und die Rolle des einen und des anderen ist wandelbar. Jedoch bleibt durch die Benutzung und Aneignung des Textes durch den Körper der Text performativ.

„Ja, also der Körper des Schauspielers ist natürlich immer was ganz, ganz Spezielles, also das ist vielleicht das Besondere, dass jeder Schauspieler auch einen ganz eigenen Körper hat, also einmal das, also es gibt den ganzen dicken oder den großen, dünnen und den erschöpften Menschen oder den sportlichen Menschen. Das heißt, er zeigt erstmal auf die vielen unterschiedlichen Arten von Körpern, die es überhaupt gibt in unserer Gesellschaft. Letztlich zeigt er aber auch, dass der Körper enorm wandelbar ist, also dass der Körper in der Lage ist, unterschiedliche Biographien in sich reinzulassen, unterschiedliche Möglichkeiten, etwas zu sein. Also der Schauspieler kann ja unterschiedliche Zustände herstellen, unterschiedliche Personen herstellen, und er zeigt doch erstmal, dass der Mensch zu unglaublich vielen Sachen eigentlich theoretisch in der Lage ist und dass oftmals der Mensch in seinem Handeln durch soziale Umstände geprägt ist, durch direkte Beziehungskonstellationen geprägt ist, von Ängsten abgehalten wird, etwas zu tun also, letztlich wird, glaube ich, über den Körper des Schauspielers für den Zuschauer eigentlich sichtbar, wie Menschen fühlen, leben, denken und wie sie 
von der Gesellschaft beeinflusst werden in ihrem Handeln. Der Körper des Schauspielers ist letztlich natürlich auch wieder das Grundmaterial des Theaters, also das Theater ist eigentlich immer auf einer Ebene ein Konzept und das trifft dann auch das Reale. Mit Konzept meine ich auch den Text oder die Inszenierungsideen oder das Konzept für den Raum. Das ist alles sozusagen noch auf der konzeptionellen Ebene und das trifft dann über den Körper des Schauspielers... wird es überhaupt erst transportiert zu den Zuschauern.

Deshalb haben wir natürlich auch so eine historische Inszenierungstradition, weil wir denken, dass jeder Hamlet in jedem Jahrzehnt immer wieder anders ist, weil die Körper ja anders sind. Also, wir haben jetzt mit dem Hamlet von Lars Eidinger einen anderen Hamlet als mit Laurence Olivier, weil die Männer anders sind heute“1.

Mit dem Beispiel von Hamlet und der Leistung des Schauspielers Lars Eidinger erklärt Falk Richter, wie der Körper des heutigen Menschen sich verändert hat und wie man gleichzeitig sich selbst als Figur engagiert, aber auch wie man die Figur an sich zieht, bzw. anzieht. Jede Rolle ist nämlich ein Teil des Schauspielers selbst und auch ein Teil der Figur, die von dem Autor erfunden wurde.

„stefan a la sensation que le monde l'a perdu

ses amis s'inquiètent parce que depuis 3 jours il n'a plus rien écrit de nouveau sur son profil facebook

que se passe-t-il, il n'a plus aucune idée

sur facebook la plupart des gens sont mieux que moi ; ils ont des phrases plus drôles, plus déjantées, des photos plus cool et ils connaissent tous emmanuelle béart et mathieu amalric et certains sont même en lien avec quentin tarantino et moi je ne connais que $20 \%$ des gens qui sont mes amis, et ce ne sont que des comédiens au chômage, des étudiants en études théâtrales ou mes parents ; et ça me donne plus ou moins la sensation d'être un loser"'2.

In diesem Ausschnitt des Textes Trust handelt es sich um den abwesenden Körper $^{3}$, um die Beziehung zur Gesellschaft, zum Anderen, zu sich selbst und um die Einsamkeit des Seins in Verbindung mit diesen verschiedenen Nicht-Beziehungen. Dieser Text stellt uns die Frage des Körpers und Fragen, die in unserer Gesellschaft besonders prägnant sind: Wie kann der Körper da sein, ohne da zu sein? Wie kann dieser in Gesellschaft, mit anderen Menschen leben, ohne zu kommunizieren? Und Wie formt sich diese Problematik zur Performativität?

1. - Interview mit Falk Richter, Theaterautor und Regisseur, Berlin, November 2011. In den nächsten Fußnotizen wird dieses Interview mit Interview mit F. R. bezeichnet.

2. - Falk Richter, Trust. Nothing hurts, L'Arche Éditeur, 2010, S. 67.

3. - Antonio R. Damasio, Le Sentiment même d'être soi, Odile Jacob, 2002. David Le Breton, L'Adieu au corps, Éditions Métailié, 1999. 


\begin{abstract}
„Wie physisch kann sich auch der Text ausdrücken. [Dann eine physische Beziehung zum Text zu finden.] Genau. Also, zum Bespiel ist das so mit Anouk [Van Dijk], dass sie ab und zu richtig meine Texte nimmt und die in eine Bewegungssprache übersetzt, das heißt sie nimmt Texte wirklich als Grundlage, um daraus eine Bewegungsabfolge, eine Bewegungsstruktur zu erfinden, und das führt auch manchmal dazu, dass ich Texte wieder rausnehme aus dem Projekt, also weil sie dann nicht als geschriebener Text vorkommen, sondern sie sind schon physisch anwesend durch die Körperlichkeit und dann nehme ich vielleicht andere Texte dazu, also dass sich dann total unterschiedliche Layer, unterschiedliche Schichten ergeben. Ja. Aber die Grundlage ist oftmals ein Text von mir, der dann auch von ihr körperlich umgesetzt wird.“
\end{abstract}

Die Fragestellung der Performativität führt uns dazu, den Vergleich mit dem Ritus anzusehen. Tatsächlich ist das Theater, ebenso wie der Ritus, durch Inszenierungen zu sehen. Diese sind gewählte, aufgebaute und wiederholte Zeitpunkte. Im Rahmen dieser Inszenierungen weiß jeder, der/die SchauspielerIn und der/die ZuschauerIn, dass die vorgeschlagene Aktion ein Spiel, also fiktiv ist. Der Ritus schöpft seine Handlungen aus der Bewegungskunst und umgekehrt. Außerdem lehnen sich beide an das alltägliche Leben an. Darin befolgen sie einen analogen Prozess, durch den die Gesten und ihre alltäglichen Bedeutungen zu einer anderen Sprache verwandelt werden. Dank der Wiederholung entsteht die Verwandlung in den nicht-alltägliche[n] Körper $^{4}$. Bei der Theaterarbeit ist die Wiederholung einer der grundlegendsten Aspekte: Diese ist von dem Prozess der Dekonstruktion, Erforschung, Konstruktion, Aufnahme, Aneignung und der Kreation untrennbar. Dadurch findet der/die SchauspielerIn den Rhythmus, der in der Gegenwart und in der Handlung verankert ist. Die Bühnenarbeit, genauso wie der Ritus, erfordert die Schöpfung einer Zeit außerhalb der alltäglichen Zeit, die aber mit dieser auch verbunden ist. Mittels dieser Hin-und Herbewegung, von der Bühne zum Leben und vom Leben zur Bühne, vertieft sich der/die SchauspielerIn in eine Art anthropologische Recherche, in der alltägliches Leben, soziokulturelle und anthropologische Fragen und schließlich auch persönliche Erfahrungen sich mischen.

Mittels seines/ihres Körpers verinnerlicht der/die SchauspielerIn die notwendige Technik, die zur angeeigneten Geste führt. Dadurch kann er/sie die Performativität erreichen. Im klassischen Begriff 5 bezieht

4. - Übersetzung von „le corps extra-quotidien“: So nennt Eugenio Barba den Körper, der für die Bühne bearbeitet wird, in Eugenio, Barba, Le Canoë de papier. Traité d'anthropologie théâtrale, Éditions L'Entretemps, 2004, p. 39.

5. - Pierre Bonte, Michel Izard, Dictionnaire de l'ethnologie et de l'anthropologie, Presses Universitaires de France, 2010, p. 630-632. 
sich der Ritus auf eine „kalte“ Eigenschaft, während die „Wärme“ zur Dauerbewegung des Lebens gehört. Darin leitet sich unser Vorschlag ab, die Performativität des Akteurs (Bühne und Riten) als Treffpunkt der Kälte und der Wärme zu sehen.

Diese Art Treffen verbinden wir mit der entwickelten Forschung Falk Richters, d.h. der Erneuerung durch das Experiment.

„Vor allem für mich, also weil ich selber ja mich, mir ist es eigentlich egal, welcher Kategorie ich bin, ja also insofern gehe ich also von dem aus was auch mich persönlich interessiert. Und mich interessiert natürlich auch die Stimme des Tänzers, weil sie nicht trainiert ist, also die Tänzer haben keine Ausbildung für die Stimme. Die Schauspieler haben eine Stimmenausbildung, das heißt im gewissen Sinne sind die auch genormt. Das ist eine normative Stimme. Sie hat vier Jahre Training gehabt. Die wissen genau, wie sie bestimmte Sachen machen, und manchmal ist es für mich interessanter, mit jemandem zu arbeiten, der gar nicht die Ausbildung hat, um zu gucken was da passiert. Oder auch zu singen, ich habe auch für Oper gemacht, also ich finde auch diesen Übergang interessant vom Sprechen zum Singen. Ich glaube, dass ich persönlich gebe... immer versuche wirklich ein Experiment, oder immer was zu untersuchen, was Neues auch zu suchen oder auch zu experimentieren eigentlich in meinen Proben mit neuen Möglichkeiten. Ich habe auch mal eine Oper gemacht für Sänger und Schauspieler, wo die Schauspieler immer mehr zum Singen kamen und die Sänger auch immer mehr gespielt haben. Das ging immer um die Verbindung zwischen Gesang und Text, ${ }^{6}$.

Nach diesem Experiment des Ausdrucks des Körpers verweist Falk Richter auf verschiedene Parameter, die den Aufbau des Körpers in der Gesellschaft gründen: Sein geschichtliches Erbe, die Familie und andere menschliche Beziehungen und schließlich nicht nur die Ausbildung, sondern auch die Erziehung.

Et si cette pièce s'appelait HISTOIRE ALLEMANDE, alors on approfondirait peut-être l'origine, l'histoire, le passé de ces parents, on s'intéresserait à leur éducation, à leurs parents, et aux parents de leurs parents, à l'Allemagne et l'Europe de cette époque, on s'attaquerait aux rapports parents-enfants CETTE RELATION DE PEUR AGRESSION ÉTRANGETÉ entre les générations, les coups que les parents portaient sur leurs enfants, les coups pour leur inculquer l'obéissance, l'ordre et la discipline PARCE QUE LA SEULE IMAGE QU'ON AVAIT DE LA SOCIÉTÉ ALLEMANDE ÉTAIT L'ARMÉE7?

6. - Interview mit F.R.

7. - Falk Richter, My Secret Garden, Paris : L'Arche Éditeur, 2010, p. 33. Kreation und Aufführung Juli 2010, Festival d'Avignon. 
Im Laufe der Aneignung der Geste in den sozialen Interaktionen, schöpft sich der Mensch eine innere Welt von Vorstellungen. Er verinnerlicht die Erfahrungen, die von außen kommen, und bringt sie zu etwas anderem. Mittels der Verinnerlichung mischt der Mensch die äußere mit der inneren Welt, um ein neues Objekt zu schaffen. Der neue Vorschlag ist auf keinen Fall eine exakte Nachahmung von dem, was er erlebt hat, sondern eine neue Schöpfung. Aus dieser neuen Version schimmern zur selben Zeit soziale Merkmale und neue Materien durch.

Was die Bühne betrifft, so besteht die theatralische Mimesis ${ }^{8}$ aus einem Umwandlungsphänomen.

„La mimésis vise le développement d'une image intérieure que le peintre ou le poète a en lui. Lors du processus artistique, quelque chose de nouveau est créé. L'esquisse qui guide la création disparaît toujours plus à l'intérieur de l'image, de la pièce de théâtre, ou du morceau de musique, qui naissent dans un autre média, que dans celui de l'esquisse imaginée. Il se produit alors des modifications, omissions, élargissements, si bien que la ressemblance reste limitée"9.

Im Bereich des theatralischen Kreierens ist der Körper als Instrument erfasst, dessen Gestik sich vom der alltäglichen Gestik ableitet. Derjenige bewegt sich dann in einer Raum-Zeit-Sphäre, wo der Körper desartikuliert wird, um die weiteste Bandbreite seiner expressiven Eigenschaften zu entdecken. Sei der gewählte Zugang psychologisch oder physisch, werden in beiden Fällen der Körper und seine Bewegungen sehr minutiös analysiert, genauso wie die vollzogenen Bewegungen und die Wirkung auf den/die SchauspielerIn und ZuschauerIn.

In einigen zeitgenössischen Formen der Exploration, die auf Improvisationen basiert sind und dem Aufbau des Stückes dienen, wie bei den écrivains de plateau ${ }^{10}$, sagt der phänomenale Körper etwas über den gegenwärtigen Augenblick. Die während der Erforschung beobachteten, gefundenen, gewählten und wiederholten Gesten werden gespeichert. Nach dieser Untersuchung sind die gewählten Gesten aus dem phänomenologischen Kontext, in dem sie erschienen sind, extrahiert, um gehalten und choreographiert zu sein. Durch diesen Prozess werden

8. - Der Begriff "theatralische Mimesis" wird in Nadia Foisil, Le Corps instrument du comédien. Gestuelle et mimésis, empreintes et vecteurs socioculturels et historiques, Dissertation, Université de Strasbourg-Freie Universität Berlin, 2012, aufgrund des Begriffs "kreative Nachahmung" nach Christoph Wulf, in Une anthropologie historique et culturelle. Rituels, mimésis sociale et performativité, Éditions Tétraèdre, 2007, p. 27, entwickelt. Siehe auch Gunter Gebauer; Christoph Wulf, Jeux, rituels, gestes. Les fondements mimétiques de l'action sociale, Éditions Economica, 2004.

9. - Christoph Wulf, "Mimésis ", in Traité d'anthropologie historique. Philosophies, histoires, cultures, L'Harmattan, 2002, p. 1051-1065.

10. - Gérard Noiriel, Histoire théâtre politique, Éditions Agone, 2009, p. 85. 
sie semiotisiert, auch wenn ihre Enderfüllung, die Endaufführung, dem/ der SchauspielerIn ein gewisses Maß an Freiheit im Zustandekommen der Geste bietet. Diese Freiheit kann sich auf den Rhythmus, die Amplitude und die Anzahl der wiederholten Gesten beziehen. Die phänomenale Geste wird semiotisiert, indem sie ihre phänomenale Dimension an dem Tag der Aufführung behält. Tatsächlich ist der/die ZuschauerIn zu dieser Aufführung als Akteur des Phänomens eingeladen. $\mathrm{Zu}$ dieser Gelegenheit bleibt der/die SchauspielerIn aufmerksam und nimmt seine Partner genauso wahr, wie er selbst wahrgenommen wird. Beide genießen die besondere Freiheit der Gesten in der öffentlichen Aufführung, während der Zuschauer auch aktiver Teilnehmer ist. Diesem Zugang gemäß, eignet sich die Mimesis ein Objekt an, verinnerlicht diesen und gibt ein neues Objekt heraus, das ergreifbar ist. Hiermit verbindet die Fremdheit dieses Objekts das Bekannte mit dem Unbekannten.

\title{
Das 21. Jahrhundert und seine Erschöpfung: ein Körper sein und einen Körper haben
}

\begin{abstract}
„Naja sagen wir's mal so, das kommt aus der Geschichte, dass wir das halt hatten. Wir hatten Brecht, der war ja noch vor der Trennung natürlich. Dann gab es in der DDR Heiner Müller, sehr wichtig. Und hier gab's ein paar andere Leute, eben Botho Strauss zum Beispiel eher, aber auch so starke, es gibt, gab in Deutschland eben immer wieder so starke, prägende Regisseure wie Einar Schleef, oder Frank Castorf zum Beispiel oder Christoph Schlingensief, die so radikale neue Theaterformen entwickelt haben. Und gleichzeitig ... ja so natürlich sieht man dann in Avignon auf den Festivals, natürlich gibt es ganz viel interessante Sachen aus anderen Ländern, das ist gar keine Frage. Ich glaube, dass sich das Nationale so langsam auflöst, also deshalb kann ich das gar nicht beschreiben, so was ist das deutsche Theater? Ich denke man wird bald von einem europäischen Theater reden. Aber natürlich ist das deutsche Theater in der Regel noch auf Text orientiert aber sehr mutig, was Experimente angeht" $" 11$.
\end{abstract}

In seinen Werken bearbeitet Falk Richter verschiedene Themen, die für ihn relevant sind, um über den aktuellen Zustand der europäischen Gesellschaft zu sprechen: Kapitalismus, Erschöpfung des Körpers, materielles und affektives Erbe, menschliche Beziehungen, usw.

Die theatralische Erfahrung und die Verwendung der Geste als Element des Körper-Instruments verweist auf das menschliche Bewusstsein. Dies erlaubt ihm, seiner Existenz, seiner Gesten und ihrer

11. - Interview mit F. R. 
Wirkung im Raum bewusst zu sein. Der Mensch ist und handelt zur selben Zeit. Diese Unterscheidung zwischen sein und haben ist mit Plessners ${ }^{12}$ Theorie bezüglich der Stelle der Menschen in der Welt eng verbunden. Gleichzeitig , ist“" er und „hat" einen Körper ${ }^{13}$.

Für den/die SchauspielerIn stellt dieses Paradox einen der grundlegenden Schwerpunkte der Frage des Körpers als professionelles Instrument dar. Dieser Körper, den er/sie wie ein für die Bühne bearbeitetes Material anwendet ist auch der, in dem und mit dem er/sie im Alltag lebt. Von diesem Dualismus leiten sich verschiedene expressive Richtungen, gemäß den theatralischen Traditionen, her. Sinn und Sensibilität werden dabei erneut entgegengestellt. Im bürgerlichen Illusionstheater des achtzehnten Jahrhunderts sticht der „semiotische Körper“ den „,phänomenalen“ aus ${ }^{14}$. Der Körper wird zum ZeichenKörper, und die Gesten werden festgesetzt und erfasst. Jede Emotion entspricht einer Ausdrucksart bzw. Ausdrucksform. Daher lernt der/ die SchauspielerIn eine kodifizierte Gestik, die den Bedürfnissen der Figur entspricht. Diese Gestik enthält eine von dem Zuschauer bekannte klare Bedeutung, die zu einer gemeinsamen Sprache gehört und die Kommunikation erlaubt. Der/die SchauspielerIn bearbeitet zum Beispiel die Bandbreite der Angst oder der Freude. "Il revient à l'acteur la tâche d'amener son corps, conformément à la règle de l'analogie, à l'expression parfaite de l'âme de son rôle. Son corps a pour seule fonction d'être un signe naturel pour les passions et les caractères, en somme pour les différents états d'une figure" 15 .

$\mathrm{Zu}$ den Bühnentraditionen, in denen der phänomenale Körper den semiotischen verdrängt oder versucht ihn zu verdrängen, gehört die Performance. In dieser Expressivitätsform, die von Erika Fischer-Lichte als « essai radical de désémiotisation conséquente du corps-acteur »16

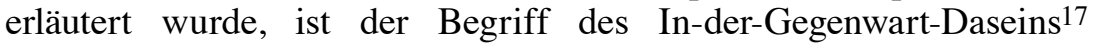

12. - Helmuth Plessner, « Conditio humana », in Gesammelte Schriften, Bd. VIII, Frankfurt/M, Suhrkamp, 1982.

13. - Erika Fischer-Lichte, «Théâtre », in Christoph Wulf, Traité d'anthropologie historique.Philosophies, histoires, cultures, 2002, p. 1019-1030. Erika Fischer-Lichte, Ästhetik des Performativen, Suhrkamp, 2004. Helmuth Plessner, « Conditio humana », in Gesammelte Schriften, Bd. VIII, Frankfurt/M, Suhrkamp, 1982. Christoph Wulf, "Mimésis », in Traité d'anthropologie historique. Philosophies, histoires, cultures, L'Harmattan, p. 1051-1065, 2002. Christoph Wulf, Une anthropologie historique et culturelle. Rituels, mimésis sociale et performativité, Éditions Tétraèdre, 2007.

14. - Erika Fischer-Lichte, «Théâtre », in Christoph Wulf, Traité d'anthropologie historique. Philosophies, histoires, cultures, L'Harmattan, 2002, p. 1019-1030. .

15. - Ebd.

16. - Ebd.

17. - Maurice Merleau-Ponty, Phénoménologie de la perception, Éditions Gallimard, 1945. Helmuth Plessner, « Conditio humana », in Gesammelte Schriften, 
verbunden mit der Ipseität. Dies konstituiert ein Fundament des Dialogs mit dem/der ZuschauerIn. In diesem Fall entfaltet der phänomenale Körper seine komplette interaktive Fähigkeit. Jedoch bleibt die Grenze zwischen den semiotischen und den phänomenalen Körpern vage. Auch wenn die Ipseitätsdimension zur Performance zu gehören scheint, gehört sie zweifellos auch zum semiotischen Körper. Schon im alläglichen Leben bestehen die Interaktionsriten ${ }^{18}$ aus einer banalisierten Performance, einer üblichen Performance. Während dieser kontinuierlichen Performances spielen sich die Szenen zwischen einem Protagonisten/einer Protagonistin und seinem/ihrem AnsprechpartnerIn ab. Diese Beziehung bildet sich sowohl auf dem was er/sie ist, als auch auf den Vorstellungen von seiner Umgebung und von was er/sie von dieser bewussten oder unbewussten Umgebung erwartet. Diese Interaktionen basieren gleichzeitig auf dem semiotischen und den phänomenalen Körper. In manchen Bedingungen bedeckt einer den Anderen, aber lässt ihn nicht komplett verschwinden. Auf der Bühne sprechen sich die beiden an.

Besser noch, sie sind eins und leiden unter dieser Dualität, die im theatralischen Milieu so wie in der Wissenschaft im Okzident prägnant ist. Die theatralische ästhetische Erfahrung ${ }^{19}$ schöpft aus dem alltäglichen Leben die Gesten, die sie benutzt, um die Kommunikation zwischen dem/der SchauspielerIn und dem/der ZuschauerIn herzustellen. Ob diese Gesten realistisch sind - oder auf eine realistische Art gespielt werden - oder stilisiert, denaturiert werden: Sie enthalten einen Sinn und vermitteln eine Bedeutung, die auf einer gemeinsamen Erfahrung basiert. Daher ist es, in dieser doppelten Art und Weise in-der-Welt-zusein (Plessner 1982), in der der Mensch sein Körper ist und sein Körper als Instrument benutzt, nötig einzusehen, was die Geste ist: Wie sie sich aufbaut, was sie bedeutet und sagt, wie man sie lernt und wie sie sich entwickelt?

„Naja und für mich persönlich ist der Körper des Schauspielers auch... Ursprung für die... also sozusagen, es ist sehr viel... es wird ein Material für meine Inszenierung, das heißt, der Körper des Schauspielers ist schon etwas, mit dem ich arbeite. Ich hatte das schon bei Protect me, da habe ich eine Improvisation gemacht mit den Schauspielern, dass sie..., die haben sich alle so in eine Reihe gestellt und dann haben sie von ihrem Körper ausgehend Geschichten erzählt. Also, wenn sie zum

Bd. VIII, Frankfurt/M, Suhrkamp, 1982.

18. - Erving Goffman, Les Rites d'interaction, Les Éditions de Minuit et Erwing Goffman, 1974.

19. - Nadia Foisil, Le Corps instrument du comédien. Gestuelle et mimésis, empreintes et vecteurs socioculturels et historiques, Thèse de Doctorat, Université de Strasbourg - Freie Universität Berlin, 2012, p. 45. 


\begin{abstract}
Beispiel, also, weiß nicht, da hatte jemand einen Autounfall und hat hier so eine Wunde, ja und fängt dann an sozusagen darüber zu erzählen; zeigt das, zeigt, was er da hat, und sagt, was da passiert ist. Er war elf Jahre alt und hatte damals einen Unfall, dabei ist er fast gestorben, und seitdem kann er den Arm nicht mehr richtig bewegen. Also so, so sozusagen so. Oder jemand hat irgendwie einen Schnitt hier, weil sein Bruder ihn da mal irgendwie attackiert hat. Und und ich hab also dann angefangen, die Leute von ihren speziellen Körpern erzählen zu lassen. Was ist die Geschichte ihres Körpers. Oder einige von diesen Geschichten sind auch... sogar in die Aufführung reingekommen“20.
\end{abstract}

Die unterschiedlichen Bewegungstechniken und Gesten, die im Theater erarbeitet werden, sind unermessliche expressive Fundamente, sind aber nicht performativ ohne ein inneres Leben, dass in sozialen Aufführungen, die von allen Menschen geteilt, aber verschieden, sogar einzigartig, aufgenommen werden. Die Begegnung der Teilnehmer einer Aufführung benötigt außerdem eine sensorisch-kommunikative Dimension, die auf verschiedenen Ebenen auftritt. Im Moment der Entdeckung einer Geste nimmt diese an einem Decodierungsprozess der Erscheinungswege der Gesten teil, aber auch an dem Auseinandernehmen der Erscheinung von Körper im Raum und von Raum im Körper. Mit den Proben, der Wiederabspielung, kommt auch das Bewusstsein und eine Verschlüsselung, die die Interventionen des Körpers im Raum und des Raumes im Körper vermischt.

Diese Verschlüsselung fixiert nicht nur die Gesten und deren originelle Form, sondern auch die unterschiedlichen Variationen, die im Laufe der Arbeit identifiziert wurden. Diese Variationen, die natürlich auch einer speziellen Zeit und einem Raum, genauso wie dem emotionellen und körperlichen Stand des Akteurs und den anderen Teilnehmern zuzuordnen sind.

Der/die SchauspielerIn nimmt diese experimentierten Nuancen in sich auf und gibt sie immer unterschiedlich wieder. Er erfindet sich ein Repertoire dieser Interaktionen und einen Katalog von möglichen Reaktionen und benutzt sie gemäß der Situation, die von der Aufführung abhängig ist. In dieser Situation wird die Geste in ihrem technischen Aspekt wiederholt bis zu der kompletten Selbstständigkeit dieser Geste, d.h. ihrer Aneignung. Der/die SchauspielerIn vermischt das „Kalte“ der ritualen Technik mit dem „Warmen“ der performativen Situation, in der er/sie mit dem Kontext und den aufführungsbezogenen Objekten interagiert. Von diesen Objekten weiß jeder, dass sie nur pure Regelung sind, eine vorbereitete Konstruktion, die eine gespielte Aktion zum Leben

20. - Interview mit F. R. 
bringt und wo SchauspielerIn und ZuschauerIn aktiv sind und das sich abspielende Phänomen unterstützen.

\section{Die Rolle des Zuschauers}

„Beim Theater ist auch der Zuschauer ganz wichtig. Der Zuschauer entscheidet auch über die Aufführung. Also, das heißt, wenn der Zuschauerraum fast leer ist und da sind fünf Leute und vier davon schlafen ein, ist die Aufführung ganz anders als wenn achthundert begeisterte Leute sitzen. Nur als Beispiel. Das ist im Kino völlig anders, also im Kino kann man manchmal auch nachts alleine sitzen, der Film ändert sich nicht dadurch. Beim Theater ändert sich aber die Spielweise der Schauspieler, die Energie, das ist eine wirkliche Interaktion, die daraus entsteht und das ist das Spezielle im Theater. Und ich glaube, um diese Sachen alle aufrecht zu erhalten, um zu begreifen, brauchen wir das Theater. Also letztlich um zu begreifen, dass wir doch immer eine sehr direkte Kommunikation miteinander brauchen. Dass wir nicht alles über kompliziertere Kommunikationsformen organisieren können, sondern dass wir doch immer sehr direkt miteinander verhandeln müssen“ 21 .

$\mathrm{Zu}$ einem didaktischen Klärungs- und Vereinfachungsziel lässt sich der Zuschauer in zwei Kategorien teilen: auf einer Seite der, der Realitätsflucht anstrebt, und auf der anderen Seite der, der sich für Kommunikation und intellektuelle Reflexivität interessiert. Diese zwei Typen sind sich einander jedoch nicht fremd, weil sie zur selben Menschlichkeit gehören. Außerdem verkörpert dieser Zuschauer durch seine Zuschauerrolle den Menschen angesichts des menschlichen Schauspielers. Das von Angesicht zu Angesicht unterstützt die Bedingungen für die Möglichkeiten der immanenten Veränderung, die zum Theater gehört, aber auch zum Phänomen und zur Menschlichkeit. Bezüglich der Begegnung schreibt Schiller: ,Wahr ist es, ihre Tendenzen widersprechen sich, aber, was wohl zu bemerken ist, nicht in denselben Objekten, und was nicht auf einander trifft, kann nicht gegen einander stossen“"22. Schiller identifiziert zwei Instinkte in der menschlichen Natur: den vernünftigen und den sinnlichen. In den Briefen an den Herzog von Augustenburg, in denen er eine Dialektik ausbreitet, entwikkelt er eine Erwägung über die ästhetische Erziehung des Menschen und folglich über die Opposition zwischen Natur und Kultur im Individuum selbst. Nach ihm gehört der vernünftige Instinkt zur Form, während der sinnliche zum Inhalt gehört. Jede dieser zwei Dimensionen existiert im Individuum, und beide versuchen zusammenzuleben. Nun braucht dieses

21. - Interview mit F. R.

22. - Friedrich Schiller, Lettres sur l'éducation esthétique de l'homme. Briefe über die ästhetische Erziehung des Menschen, Éditions Aubier/Domaine allemand bilingue, 1992, p. 192. 
Zusammenleben Grenzen, die den Raum der Instinkte umgrenzen und in denen die Entwicklung von jedem Instinkt das Aneignen der gegenständigen Tendenz verlangt.

Diese in den Theatertraditionen beobachteten Tendenzen geben nur ein zweiteiliges Bild des Prozesses der Theaterkreation, sind aber in Realität nicht so scharf voneinander trennbar. Genauso steht es mit dem/ der SchauspielerIn und dem/der ZuschauerIn. Der/die SchauspielerIn, der sich nur auf Technik konzentriert und sich selbst nicht Zugang zu Wahrnehmung und Konstruktion zulässt, ist kein "komplettes" Wesen, kein ganzes Wesen. Sobald der/die ZuschauerIn ein ganzes Wesen ist, ist er nicht mehr nur "Nachdenker" oder "Wahrnehmer". Diese beiden Arten der Wahrnehmung gehören dem Körper, und Körper wachsen zusammen, lernen sich kennen, unterstützen sich, widersprechen sich, tauschen, verändern sich und produzieren eine Aktion, die zu einer Interaktion wird. In dieser Zugangsweise, ist das Totale Theater ein Analyst und Wahrnehmer der Welt, die ihn umgibt; aber auch der Träger der Reaktionen auf diese Analysen und Wahrnehmungen, die sich mit dem Existenten befassen. Er ist eine Verknüpfung zwischen Räumen und Zeiten, um sie schließlich in einem kontinuierlichen Prozess zu vereinen. Dieser Prozess läuft natürlich über den/die SchauspielerIn, der ihn dem Zuschauer weitergibt, der selbst diese neuen Daten mit seinen schon vorhandenen Daten konfrontiert, um diesen Austausch in eine Kreisbewegung zu bringen; Kreisbewegung, die das Theater zum Leben erweckt. So ist es nun schwierig, den "Nachdenker" von dem "Wahrnehmer" zu unterscheiden. Natürlich gibt es bei jedem Menschen eine unterschiedliche Dosis dieser Eigenschaften, und jede Etüde, die diese Faktoren auslassen würde, wäre nicht vollständig und nähme dem Zuschauer ein Stück Menschlichkeit davon. Außerdem ist der/die ZuschauerIn, der/die Fragen, eine Problematik, im Theater aufkommen lässt, seiner/ihrer Sinne nicht beraubt. Er/sie bleibt ein organisches Wesen in Beziehung zu Menschen, selbst Akteure der Prozesse, die er/sie analysiert und in denen er/sie selbst SchauspielerIn ist.

Als Schluss zu diesem Artikel tauchen wieder die Überlegungen über die Merkmale auf, die das postdramatische Theater ${ }^{23}$ charakterisieren, und die Frage, ob Falk Richters Werke zum postdramatischen Theater gehören. "[...] mir ist es eigentlich egal, welcher Kategorie ich bin, ja also insofern gehe ich also von dem aus, was auch mich persönlich interessiert." 24 Und eigentlich ist es schwierig, diese Werke in

23. - Lehmann Hans-Thies, Le Théâtre postdramatique, L'Arche Éditeur, 2002.

24. - Interview mit F. R. 
einer Kategorie aufzuräumen. Bald ist er neodramatisch ${ }^{25}$, bald postdramatisch. Wie er es behauptet, löst sich das Nationale auf, und man kann eher von einem europäischen Theater sprechen, aber auch ist das deutsche Theater "[...] noch auf Text orientiert, aber sehr mutig, was Experimente angeht" 26 .

Barba, Eugenio, Le Canoë de papier, Traité d'Anthropologie Théâtrale, SaintJean-de-Védas, Éditions L'Entretemps, 2004.

Damasio, Antonio R., Le Sentiment même d'être soi, $2^{\mathrm{e}}$ éd., Paris, Odile Jacob, 2002.

Fischer-Lichte, Erika, Ästhetik des Performativen, Frankfurt am Main, Suhrkamp Verlag, 2004.

Gebauer, Gunter und Wulf, Christoph, Jeux, rituels, gestes. Les fondements mimétiques de l'action sociale, Paris, Éditions Economica, 2004.

Goffman, Erving, Les Rites d'interaction, Paris, Les Éditions de Minuit et Erwing Goffman, 1974.

Le Breton, David, L'Adieu au corps, Paris, Éditions Métailié, 1999.

Lehmann, Hans-Thies, Le Théâtre postdramatique, Paris, L'Arche Éditeur, 2002.

Merleau-Ponty, Maurice, Phénoménologie de la perception, Paris, Éditions Gallimard, 1945.

Noiriel, Gérard, Histoire Théâtre Politique, Marseille, Éditions Agone, 2009.

Plessner, Helmuth, « Conditio humana », in Gesammelte Schriften, Frankfurt/M, Suhrkamp, VIII, 1983.

Schiller, Friedrich, Lettres sur l'éducation esthétique de l'homme. Briefe über die ästhetische Erziehung des Menschen, Paris, Éditions Aubier/Domaine allemand bilingue, 1992 .

Wulf, Christoph, Traité d'anthropologie historique. Philosophies, histoires, cultures, L'Harmattan, 2002.

Wulf, Christoph, Une anthropologie historique et culturelle. Rituels, mimésis sociale et performativité, Paris, Éditions Tétraèdre, 2007.

25. - Kabale und Liebe von Friedrich Schiller, Inszenierung von Falk Richter, Schaubühne Berlin, 2010.

26. - Interview mit F. R. 
